patients continued to undergo outpatient rehabilitation treatment after the 90 day follow-up visit. Mean time to longterm clinical follow-up was 21.5 months after stroke onset (median 18.8 months, range 6-49 months). The table illustrates the clinical outcomes at the time of long-term followup. Between the 90 day and long-term follow-ups, 12 patients (30\%) had a reduction of at least 1 point in the mRS, whereas 10 patients $(25 \%)$ had an increase of at least 1 point in the mRS. At the time of long-term follow-up, 23 patients lived at home (58\%), 3 in an assisted living facility (8\%), 6 in a skilled nursing facility (15\%) and 8 were deceased (20\%). Four patients had experienced a recurrent AIS (10\%) by the time of long-term follow-up.

Conclusion Among patients with AIS treated with MT who had moderate or moderate severe disability at 90 days, a considerable minority $(30 \%)$ demonstrated a reduction in longterm disability with continued outpatient rehabilitation.

\begin{tabular}{|c|c|c|c|c|c|}
\hline \multicolumn{6}{|c|}{$\begin{array}{l}\text { Long-term outcomes in Pts w moderate or moderate severe disability after } \\
\text { mechanical thrombectomy }\end{array}$} \\
\hline & \multicolumn{5}{|c|}{ Long-term modified rankin scale } \\
\hline & 2 & 3 & 4 & 5 & 6 \\
\hline \multirow[t]{2}{*}{ All Patients $(n=40)$} & 5 & 18 & 7 (17.5\%) & 2 & 8 \\
\hline & $(12.5 \%)$ & $(45 \%)$ & & $(5 \%)$ & $(20 \%)$ \\
\hline Patients with Moderate Disability & 4 & 11 & 0 & 0 & 3 \\
\hline at 90 Days (mRS $3, \mathrm{n}=18$ ) & $(22.2 \%)^{*}$ & $(61.1 \%)$ & & & $(16.7 \%)$ \\
\hline Patients with Moderate Severe & 1 & 7 & 7 & 2 & 5 \\
\hline Disability at 90 Days & $(4.5 \%)^{*}$ & $(31.8 \%)^{*}$ & $(31.8 \%)$ & $(9.1 \%)$ & $(22.7 \%)$ \\
\hline$(m R S 4, n=22)$ & & & & & \\
\hline
\end{tabular}

Disclosures J. Delgado Almandoz: 2; C; Medtronic Neurovascular, Penumbra, Inc. S. Hochsprung: None. Y. Kayan: 2; C; Medtronic Neurovascular, Penumbra, Inc. J. Fease: None. J. Scholz: None. A. Milner: None. P. Roohani: None. M. Mulder: None. T. Hehr: None. R. Tarrel: None. D. Chappuis: None. M. Young: None.

\section{E-085 GOOD BASELINE ANGIOGRAPHIC COLLATERAL FLOW CORRELATES WITH SUCCESSFUL RECANALIZATION AND CLINICAL OUTCOME AFTER ENDOVASCULAR TREATMENT FOR ACUTE ISCHEMIC STROKE WITHIN 24 HOURS FROM ONSET}

1J Seo, ${ }^{1} \mathrm{E} \mathrm{Kim},{ }^{2} \mathrm{H}$ Jeong. ${ }^{1}$ Neurology, Inje University colleage of Medicine, Busan, Republic of Korea; ${ }^{2}$ Radiology, Inje University colleage of Medicine, Busan, Republic of Korea

\subsection{6/neurintsurg-2016-012589.157}

Background Recanalization status is the strongest predictor of clinical outcome in patients undergoing endovascular treatment (EVT). Although the time to reperfusion remains crucial even with EVT, the previous meta-analysis demonstrated that recanalization up to 24 hours after acute ischemic stroke (AIS) onset is strongly associated with improved functional outcomes and reduced mortality. We evaluated the relationships among angiographic collateral flow, successful recanalization, and clinical outcome after EVT in patients experiencing AIS within 24 hours of onset.
Methods We assessed patients were experiencing acute anterior circulation ischemic stroke who underwent EVT between 2011 and 2015. Patients with large artery occlusion of anterior circulation and clinical diagnosis of AIS, within 24 hours of first found abnormal time (FAT), were included in the study.

Results One hundred seventy-three patients met the inclusion criteria. Mean age was $68.3 \pm 12.6$ years, and median National Institutes of Health Stroke Scale score was 14 (range, 5-29). Median time from FAT to arrival was 92 minutes. Overall successful recanalization, defined by the Modified Thrombolysis In Cerebral Infarction scale grade 2 b-3, was achieved in $65.3 \%$ of patients, and good clinical outcome, as defined by the modified Rankin Scale (mRS 0-2), was achieved in $47.4 \%$ of patients. For successful $r$ the angiographic collateral grade was the independent factor. In multiple logistic regression analysis, the angiographic collateral grade was independently associated with clinical outcome after adjusting for other variables (odds ratio, 5.96; 95\% CI, 1.7620.19).

Conclusions Our data showed that angiographic collateral grade was a strong independent predictor of successful recanalization after EVT and good clinical outcome in AIS patients when applied up to 24 hours from FAT. Consequently, the good angiographic collateral flow may help predict successful recanalization and better clinical outcomes after EVT in patients with AIS.

Disclosures J. Seo: None. E. Kim: None. H. Jeong: None.

\section{E-086 MANDIBULAR AVM DIAGNOSIS AND CURATIVE TREATMENT}

W Yakes. Vascular Malformation Center, Englewood, CO

\subsection{6/neurintsurg-2016-012589.158}

Purpose To determine optimal management strategies for the treatment of mandibular AVM.

Materials and methods Twelve patients (9 females, 3 males), age 9-14; mean age 10, underwent endovascular therapy to treat their mandibular AVMs. Nine patients had distinct intraosseous AVMs. Three had multiple facial and intra-maxillary AVMs requiring treatment. Outside institutions recommended massive hemi-facial resections in these patients. Four patients had prior PVA and gel foam embolization, one patient had a lip graft, one had prior mandible surgery, all that had failed.

Results All twelve patients have demonstrated MR and angiographic cure of their AVMs. One patient's therapy is not completed and is on-going. The patients mandibular AVM is cured, a third AVM in this patient in the infratemporal fossa is still undergoing treatment. The follow-up range is 11 months - 41 months, with a mean follow-up of 29 months. No complications were noted in treatment of mandibular AVMS. One patient required a minor gingival surgery after treatment of an additional intramaxillary AVM.

Conclusions Endovascular approaches to manage mandibular AVM can be curative. The intraosseous variety is largely a fistula between artery and vein within the bone. All respond well to endovascular ethanol therapy alone. Surgery was not required in any patient. Surprisingly no complications were encountered in this patient series. Long-term cures are noted in this patient series with endovascular approaches alone. No massive surgical resections in any patient, even in patients with multiple AVMs of the soft tissues, mandible and maxilla, 